\title{
Two-dimensional Bose polaron using diffusion Monte Carlo method
}

\author{
E. Akaturk* and B. Tanatar ${ }^{\dagger}$ \\ Department of Physics, Bilkent University, Bilkent 06800 Ankara, Turkey \\ *akaturk@fen.bilkent.edu.tr \\ ${ }^{\dagger}$ tanatar@fen.bilkent.edu.tr \\ Received 19 April 2019 \\ Revised 16 June 2019 \\ Accepted 5 July 2019 \\ Published 5 September 2019
}

\begin{abstract}
We investigate the properties of a mobile impurity immersed in a two-dimensional (2D) Bose gas at zero temperature using quantum Monte Carlo (QMC) methods. The repulsive boson-boson and impurity-boson interactions are modeled by hard-disk potentials with positive scattering lengths $a$ and $b$, respectively, taken to be equal to the scattering lengths. We calculate the polaron energy and effective mass for the density parameter $n a^{2} \ll 1$ and the ratio $a / b$. We find that at low densities perturbation theory adequately describes the simulation results. As the impurity-boson interaction strength increases, the polaron mass is enhanced. Additionally, we calculate the structural properties of the Bose system, such as the impurity-boson pair-correlation function and the change of the density profile around the impurity.
\end{abstract}

Keywords: Bose polaron; impurity-boson interactions; two dimensions.

PACS numbers: 03.75.Hh, 02.70.Ss, 67.60.Bc, 31.15.ac

\section{Introduction}

An electron interacting with the vibrational modes of atoms in a crystal is called a polaron..$^{-\underline{5}}$ This quasiparticle which is made up of the electron surrounded by a cloud of phonons has physical properties such as binding energy (polaron energy) and effective mass that are quite different than that of the bare electron. About 30 years ago, polarons and bipolarons came to the fore in the context of high critical temperature (high- $T_{c}$ ) superconductivity $\underline{\underline{6}-\underline{8}}$ and they were instrumental in our understanding of various many-body systems such as organic superconductors and heavy-fermion superconductors.

Recently, ultracold atomic systems have also been very fruitful in simulating condensed matter phenomena and there has been a large amount of recent experimental and theoretical effort to investigate polarons in these systems. A

\footnotetext{
* Corresponding author.
} 
number of experiments considered impurity atoms in Bose-Einstein condensates $(\mathrm{BEC})^{9-12}$ paving a way to two recent works, in which an atomic implementation of polaronic physics was demonstrated. ${ }^{13,14}$ The impurity interacts with the condensate similar to an electron interacting with surrounding atoms in a crystal. By tuning the strength of interactions, it was possible in these recent experiments to access the regime of strong coupling between the impurity and the condensate resulting from many-body correlations. Earlier works studied the Fermi polaron in detail which is an impurity surrounded by fermionic atoms. $\frac{15}{}$ New experiments (our main motivation for this work) used Bose condensed gases and an impurity atom therefore the quasiparticle forming in these new systems are called Bose polarons.

There has been a large number of theoretical investigations addressing the polarons in BECs. Astrakharchik et al. $\frac{16}{}$ studied the motion of a point-like impurity by solving Gross-Pitaevskii equation. Self-localization of polarons were studied by a number of groups ${ }^{17-23}$ employing methods ranging from mean-field theory to Feynman's variational approach. T-matrix approach is used by Rath et al. ${ }^{24}$ to calculate quasiparticle properties and by Volosniev et al. $\frac{25}{}$ to study the behavior of a harmonically trapped system. Li et al. $\frac{26}{}$ developed a variational approach and Christensen et al. ${ }^{27}$ investigated quasiparticle properties upto third order in perturbation theory. Three body effects (i.e., Efimov effect) were studied by Levinsen et $a l .28$ and it was shown that they lower the energy significantly. Grusdt et al. ${ }^{29}$ used renormalization group approach to analyze the problem. Very recently, Pastukhov ${ }^{30}$ investigated the properties of a Bose polaron in a dilute $2 \mathrm{D}$ system at low-temperatures.

On the numerical side, Ardila and Giorgini ${ }^{31,32}$ studied the Bose polaron using diffusion Monte Carlo (DMC) simulations to calculate quantities such as the polaron energy, polaron effective mass and the density profile of particles around the impurity. In the case of lower dimensions, there has been a QMC study of the Bose polaron system in $1 \mathrm{D}$ by Parisi and Giorgini ${ }^{33}$ and Grusdt et al. ${ }^{34}$ Other theoretical works in lower dimensions include the study of self-localization in a quasi-1D BEC by Sacha et al. ${ }^{35}$ and investigation of a driven impurity in a 1D Bose gas by Castelnovo et al. ${ }^{36}$ Bose polarons in a dipolar gas have also been considered. ${ }^{37}$

Motivated by the above experimental and theoretical works, in this paper, we report our QMC simulations of a system of $2 \mathrm{D}$ bosons and a single mobile impurity. To our knowledge there is no realization of a Bose polaron in 2D, but recently Grusdt and Fleischhauer ${ }^{38}$ suggested an experimental setup in a quasi-2D BEC system. Using hard-disk potentials to describe the short-range interactions between bosons and the impurity and bosons among themselves, we perform DMC calculations of the polaron energy and effective mass for a range of boson-impurity interaction strengths.

The rest of this paper is organized as follows. In Sec. 2, we briefly introduce the physical model we are investigating and provide basic equations from the perturbation theory. We summarize the DMC method in Sec. 3. Results of our simulations and their discussion are given in Sec. 4 . We conclude with some remarks in Sec. 5. 


\section{Model and Perturbative Results}

Our model consists of a single impurity immersed in a $2 \mathrm{D}$ dilute Bose gas of $N$ particles at $T=0$. The Hamiltonian of the system is given by

$$
H=-\frac{\hbar^{2}}{2 m_{B}} \sum_{i=1}^{N} \nabla_{i}^{2}+\sum_{i<j} V\left(\mathbf{r}_{i}-\mathbf{r}_{j}\right)-\frac{\hbar^{2}}{2 m_{I}} \nabla_{\alpha}^{2}+\sum_{i<j} V\left(\mathbf{r}_{i}-\mathbf{r}_{\alpha}\right) .
$$

Here, the first term gives the kinetic energy of the bosons and the second term is the potential energy between them. Third term gives the kinetic energy for the impurity and the last term is the potential energy between the impurity and bosons, $m_{B}$ and $m_{I}$ are the masses of the bosons and the impurity, respectively. Interaction potentials are modeled as hard-disks. More explicitly, the boson-boson and impurity-boson interactions are given as

$$
\begin{aligned}
& V_{B}(r)=\left\{\begin{array}{cc}
+\infty, & r \leq a, \\
0, & r>a,
\end{array}\right. \\
& V_{I}(r)=\left\{\begin{array}{cc}
+\infty, & r \leq b, \\
0, & r>b,
\end{array}\right.
\end{aligned}
$$

where $a$ and $b$ are the 2D scattering lengths (corresponding to hard-disk diameters) characterizing the boson-boson and impurity-boson interactions, respectively.

To compare our simulation results, we review the perturbation theory approach to the system of a mobile impurity in a Bose gas. The Bogoliubov Hamiltonian reads

$$
H_{B}=E_{B}+\sum_{\mathbf{k}} \epsilon_{k} \alpha_{\mathbf{k}}^{\dagger} \alpha_{\mathbf{k}}
$$

where $E_{B}$ is the ground state energy of a $2 \mathrm{D}$ system of hard-core bosons ${ }^{39,40}$

$$
E_{B}=\frac{\hbar^{2}}{2 m_{B}} \frac{4 \pi n}{\ln \left(1 / n a^{2}\right)} N
$$

with $n=N / A$ the density of bosons and $\alpha_{\mathbf{k}}^{\dagger}$ and $\alpha_{\mathbf{k}}$ are creation and destruction operators for Bogoliubov quasiparticles.

Interaction energy of the impurity at position $\mathbf{r}_{\alpha}$ with the boson bath at meanfield level is given by

$$
H_{\mathrm{int}}=g_{I B} \int d \mathbf{r} n(\mathbf{r}) \delta\left(\mathbf{r}-\mathbf{r}_{\alpha}\right)
$$

where $g_{I B}=\frac{2 \pi \hbar^{2}}{m_{\mathrm{red}}\left|\ln \left(n b^{2}\right)\right|} 41$ is the coupling between the impurity and bosons with $m_{\text {red }}$ the reduced mass (i.e., $1 / m_{\text {red }}=1 / m_{I}+1 / m_{B}$ ). The above Hamiltonian can be written in momentum space using the Bogoliubov approximation $\sum_{\mathbf{k}} a_{\mathbf{k}}^{\dagger} a_{\mathbf{k}}+\mathbf{q} \simeq$ $\sqrt{n_{0} A}\left(u_{q}+v_{q}\right)\left(\alpha_{\mathbf{q}}+\alpha_{-\mathbf{q}}^{\dagger}\right)$ (in which $n_{0}$ is the density of the condensed particles, $A$ the area, and $u_{q}$ and $v_{q}$ are coefficients in the Bogoliubov transformation)

$$
H_{\mathrm{int}}=g_{I B} n+\frac{g_{I B}}{\sqrt{A}} \sum_{\mathbf{q} \neq 0} e^{i \mathbf{q r}_{\alpha}} \sqrt{n_{0}}\left(u_{q}+v_{q}\right)\left(\alpha_{\mathbf{q}}+\alpha_{-\mathbf{q}}^{\dagger}\right)
$$


which is in the form of Fröhlich Hamiltonian. ${ }^{-3}$ To leading order in perturbation theory, the ground-state energy of a system of $N$ bosons and one impurity is

$$
E_{0}=E_{B}+g_{B I} n=E_{B}+\frac{2 \pi n \hbar^{2}}{m_{\mathrm{red}}\left|\ln \left(n b^{2}\right)\right|} .
$$

Thus, the polaron energy is given by

$$
E_{P}=E_{0}-E_{B}=\frac{2 \pi n \hbar^{2}}{m_{\mathrm{red}}\left|\ln \left(n b^{2}\right)\right|} .
$$

Recent work of Pastukhov ${ }^{30}$ extended the above expression to a higher order in perturbation theory

$$
E_{P}=\frac{2 \pi n \hbar^{2}}{m_{\mathrm{red}}\left|\ln \left(n b^{2}\right)\right|}\left[1-\frac{\ln \left|\ln \left(n a^{2}\right)\right|}{\left|\ln \left(n b^{2}\right)\right|}+\frac{2 \gamma+\ln \pi+\frac{2 \alpha}{\alpha-1} \ln \frac{2 \alpha}{\alpha+1}}{\left|\ln \left(n b^{2}\right)\right|}\right],
$$

in which $\gamma=0.57721, \ldots$ is the Euler-Mascheroni constant and $\alpha=m_{I} / m_{B}$.

\section{DMC Method}

We use DMC which is a method for calculating the exact ground-state energy of a bosonic many-body system. The method aims to solve the Schrödinger equation in imaginary time $(\tau=i t)$, using a distribution function $f(\mathbf{R}, \tau)=\phi(\mathbf{R}) \psi(\mathbf{R}, \tau)$. Here, $\phi(\mathbf{R})$ is a trial wavefunction and $\psi(\mathbf{R}, \tau)$ is the exact wavefunction of the system. Imaginary time Schrödinger equation is given $\operatorname{as}^{31,42,44,45}$

$$
\frac{\partial f(\mathbf{R}, \tau)}{\partial \tau}=\frac{\hbar^{2}}{2 m} \nabla^{2} f(\mathbf{R}, \tau)-\nabla \cdot[\mathbf{V}(\mathbf{R}) f(\mathbf{R}, \tau)]-\left[E(\mathbf{R})-E_{R}\right] f(\mathbf{R}, \tau),
$$

where $E_{R}$ is the reference energy, $E(\mathbf{R})=\hat{H} \phi(\mathbf{R}) / \phi(\mathbf{R})$ is the local energy which denotes the current energy of the configuration $\mathbf{R}$ and

$$
\mathbf{V}(R)=\frac{\hbar^{2}}{m} \nabla \ln |\phi(\mathbf{R})|,
$$

is a term that can be interpreted as a drift velocity of the distribution in the configuration space. Time evolution of $f(\mathbf{R}, \tau)$ is given as

$$
f\left(\mathbf{R}^{\prime}, \tau+\Delta \tau\right)=\int G\left(\mathbf{R}^{\prime}, \mathbf{R} ; \Delta \tau\right) f(\mathbf{R}, \tau) d \mathbf{R}
$$

where the Green's function ${ }^{45}$

$$
G\left(\mathbf{R}^{\prime}, \mathbf{R} ; \Delta \tau\right)=\left(\frac{1}{2 \pi \chi^{2}}\right)^{N} e^{-\left[\mathbf{R}^{\prime}-\mathbf{R}-\Delta \tau \mathbf{V}(\mathbf{R})\right]^{2} / 2 \chi^{2}-\Delta \tau\left[E(\mathbf{R})-E_{R}\right]}
$$

is the propagator that carries the system from $\mathbf{R}$ at time $\tau$ to $\mathbf{R}^{\prime}$ at time $\tau+\Delta \tau$ and $\chi^{2}=\hbar^{2} \Delta \tau / m$.

DMC algorithm makes $f(\mathbf{R}, \tau)$ evolve through $\tau$ using to the propagator given in Eq. (12). During a DMC simulation, if the trial wavefunction $\phi(\mathbf{R})$ has some 
overlap with the true wavefunction, then $f(\mathbf{R}, \tau)$ evolves to the ground-state of the system.

Our trial wavefunction is of Jastrow type with the Jastrow factor given by the two-body scattering solution in $2 \mathrm{D}$. The trial wavefunction for the bosonic system with impurity is given by $\frac{31}{\text { : }}$

$$
\Psi_{T}\left(\mathbf{r}_{1}, \ldots, \mathbf{r}_{\mathbf{N}}\right)=\prod_{i=1}^{N} f_{I}\left(\left|\mathbf{r}_{\mathbf{i}}-\mathbf{r}_{\alpha}\right|\right) \prod_{j<k}^{N} f_{B}\left(\left|\mathbf{r}_{\mathbf{j}}-\mathbf{r}_{\mathbf{k}}\right|\right),
$$

where $f_{I}(r)$ describes the impurity-boson interaction and $f_{B}(r)$ describes the boson-boson interaction and $r_{\alpha}$ is the position of the impurity. For both interactions we use Jastrow factors of the same form. The only difference between them is the scattering length (which is used to fix the constants) as the boson-boson interaction and impurity-boson interaction has different scattering lengths. More explicitly, the Jastrow factor is expressed as

$$
f_{B, I}(r)= \begin{cases}0, & 0<r \leq a, b, \\ A J_{0}(k r)+B Y_{0}(k r), & a, b<r<d, \\ 1, & r \geq d,\end{cases}
$$

which is the solution to the two-body problem in 2D for HD potential for $r>a$ or $b .{ }^{42}$ Here, $J_{0}(x)$ and $Y_{0}(x)$ are zeroth order Bessel functions of the first and second kinds, respectively. The constants $A$ and $B$ can be fixed by imposing the boundary conditions: $f(r \leq a)=0$ (for boson-boson interaction), $f(r \leq b)=0$ (for impurityboson interaction), $f(r \geq d)=1, f^{\prime}(r \geq d)=0$ where $d$ is left as a variational parameter and it is noted to give an optimal result when $d=L / 2 . \underline{42}$ The last constraint

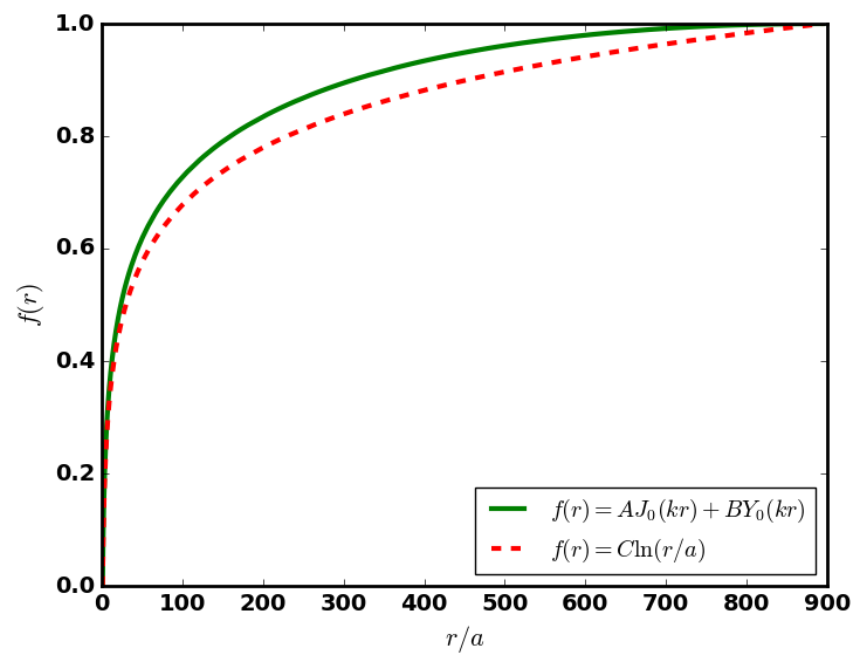

Fig. 1. (Color online) Jastrow factors for two models of hard-core interaction used in simulations as a function of $r / a$ for $r \leq L / 2$ and $n a^{2}=10^{-5}$. 
for the Jastrow factor is that only one node is allowed for $a \leq r \leq d$ (or $b \leq r \leq d$ for impurity-boson interaction). We have also employed the following Jastrow factor:

$$
f_{B, I}(r)=\left\{\begin{array}{lc}
0, & 0<r \leq a, b, \\
C \ln (r /(a \text { or } b)), & a, b<r<d, \\
1, & r \geq d,
\end{array}\right.
$$

in which $C$ is a constant determined by similar conditions above. This latter form is motivated by the recent proposal of Petrov and Astrakharchick. ${ }^{43}$ We display in Fig. 1 the two Jastrow factors used in this work at $n a^{2}=10^{-5}$ as a function of $r$ for $r<L / 2$.

\section{Results and Discussion}

We have carried out DMC simulations of systems of $N$ bosons and a single impurity using periodic boundary conditions with a square simulation box whose size $L$ is fixed by the density $n$ and by the total number of atoms $n=N / L^{2}$. We performed calculations for the density parameter $n a^{2}=10^{-5}$ and various values of the ratio of boson-boson and impurity-boson scattering lengths $a / b$. Typically the number of bosons were $N=512$, but simulations for boson numbers upto $N=1024$ were also performed to test the finite-size effects. The DMC energies are obtained from ${ }^{45}$

$$
E(\tau)=\frac{\sum_{i=1}^{N_{E}} E\left(\mathbf{R}_{i}\right) W_{B}\left(\mathbf{R}_{i}\right)}{\sum_{i=1}^{N_{E}} W_{B}\left(\mathbf{R}_{i}\right)},
$$

where $\mathbf{R}_{i}$ are the sample configurations in the ensemble and $W_{B}(\mathbf{R})=$ $e^{-\Delta \tau\left[E(\mathbf{R})-E_{R}\right]}$ is the weight of the configuration.

As the polaron energy is calculated by the difference in the energy of a system with $N$ bosons with and without an impurity, we have first reproduced the groundstate energy results for a uniform $2 \mathrm{D}$ system of bosons. $\frac{42}{2}$ Our results for the polaron energy $E_{P}$ are shown in Fig. 2 as a function of $-\ln (a / b)$ the ratio of boson-boson and impurity-boson scattering lengths for the density parameter $n a^{2}=10^{-5}$. For simplicity we have taken the boson and impurity masses to be the same. We have employed two forms of the hard-core Jastrow factor discussed above and found that the resulting polaron energy is largely independent of our choice. The influence of strong impurity-boson interaction is evident at small values of $a / b$ resulting in an enhanced polaron energy. We find that both the leading order perturbation theory and the recent expression of Pastukhov ${ }^{30}$ for $E_{P}$ agrees with simulation results at this low value of the density parameter. However, as the density parameter $n a^{2}$ is increased deviation from DMC results would become significant.

Effective mass of the impurity (polaron mass) can be found by calculating the long term slope of the mean square displacement of impurity in imaginary time. $\stackrel{46,47}{ }$ It is calculated in $2 \mathrm{D}$ using the following expression:

$$
\frac{m}{m^{*}}=\lim _{\tau \rightarrow \infty} \frac{\left\langle\left|\Delta \mathbf{r}_{\alpha}(\tau)\right|^{2}\right\rangle}{4 D \tau},
$$




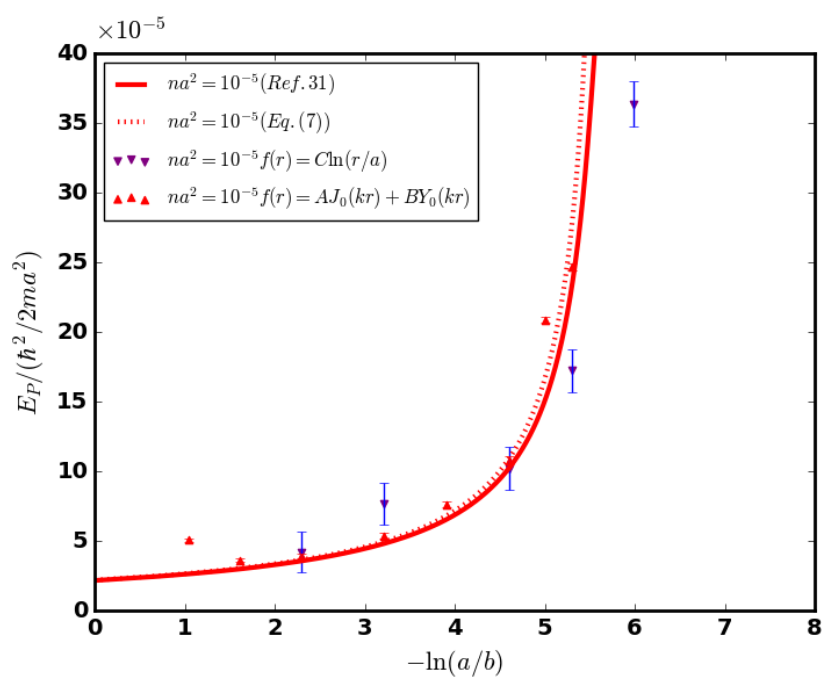

Fig. 2. (Color online) Polaron energy $E_{P}$ as a function of $-\ln (a / b)$ for the density parameter $n a^{2}=10^{-5}$. Error bars indicate statistical errors in the DMC simulations. Dashed and solid lines are the perturbation theory results from Eqs. (7) and (8) Ref. 31, respectively.

where $D=\hbar^{2} / 2 m$ is the diffusion constant of a free particle and $\left\langle\left|\Delta \mathbf{r}_{\alpha}(\tau)\right|^{2}\right\rangle=$ $\left\langle\left|\mathbf{r}_{\alpha}(\tau)-\mathbf{r}_{\alpha}(0)\right|^{2}\right\rangle$ is the mean square displacement.

We start the DMC simulation for effective mass calculation with 10 walkers, the same number we use for the other calculations. These 10 walkers, due to nature of the DMC algorithm multiply in size and reach around 1000 walkers. Number of time steps needed for the walker size to reach around the desired value is around 100. This value changes according to the different physical parameters of the DMC simulation (for example density, impurity-boson interaction strength, etc.) but not drastically. We make sure that this period is short relative to the total simulation time. Our simulations for this kind of procedure usually takes 1000 time steps. The convergence of the DMC algorithm is monitored by checking the root mean square of the impurity position in imaginary time. After large enough imaginary time steps, we calculate the effective mass using the accumulated data. A dataset comprises of around 1000 walkers each containing a copy of the physical system (with one impurity and usually 512 bosons) which has been subject to DMC algorithm for at least 1000 imaginary time steps. We display in Fig. 3 a typical time evolution of mean square displacement of the impurity atom as a function of the time step $\Delta \tau$.

We have calculated the polaron effective mass at $n a^{2}=10^{-5}$ as a function of $-\ln (a / b)$. The results of our calculations are shown in Fig. 4 . We find that $m^{*} / m$ displays the influence of strong impurity-boson interaction at large values of $b$. The perturbation theory expression of Pastukhov ${ }^{30}$

$$
\frac{m}{m^{*}}=1-\frac{1}{2 \alpha} \frac{\ln \left(n a^{2}\right)}{\ln ^{2}\left(n b^{2}\right)}
$$




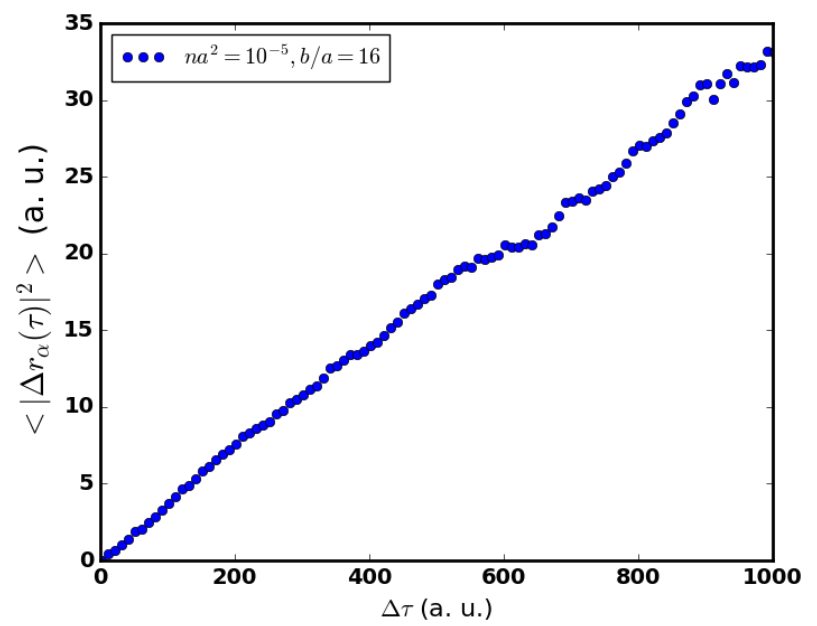

Fig. 3. (Color online) A typical mean square displacement of the impurity atom as a function of time step in arbitrary units at $n a^{2}=10^{-5}$ and $b / a=16$.

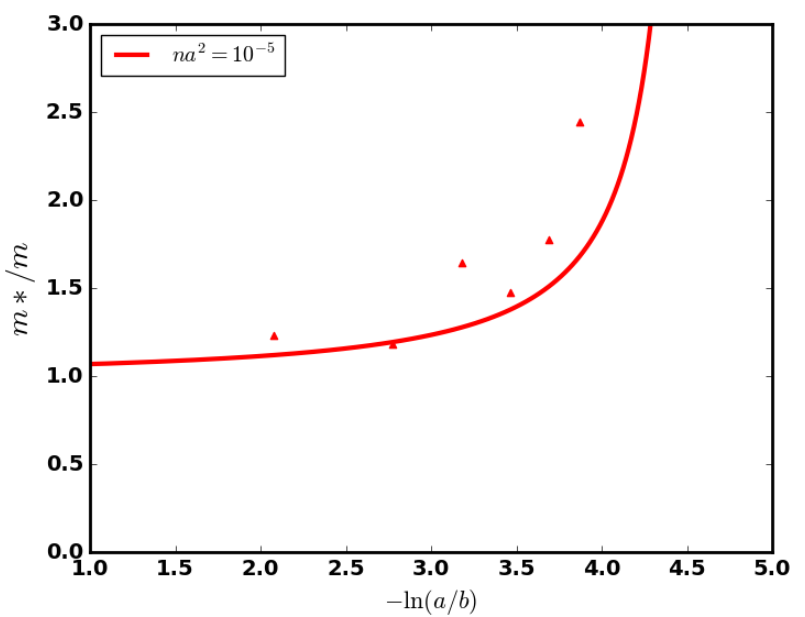

Fig. 4. (Color online) Polaron effective mass $m^{*} / m$ as a function of $-\ln (a / b)$ for the density parameter $n a^{2}=10^{-5}$.

adequately describes the trend of our simulation results. Ardila and Giorgini ${ }^{31}$ in their simulations of a 3D Bose gas with impurity found that $m^{*} / m$ remains finite approaching the resonant point $a / b=0$ implying the absence of self-localization. We also find an enhancement in $m^{*} / m$ as $a / b \rightarrow 0$ but the maximum value reached depends on the density. Furthermore, for a given density parameter $n a^{2}$ it becomes difficult to obtain converging results below a certain value of $a / b$ (not shown). Whether this indicates an instability in the system or self-localization of the impurity should be investigated further. 
We now turn our attention to the structural properties of the system at hand. Pair-correlation function gives the probability of finding a particle at a given distance and it is defined as

$$
g\left(\left|\mathbf{r}_{2}-\mathbf{r}_{1}\right|\right)=\frac{N(N-1)}{n^{2}} \frac{\int|\psi(\mathbf{R})|^{2} d r_{3} \ldots d r_{N}}{\int|\psi(\mathbf{R})|^{2} d \mathbf{R}} .
$$

In order to calculate the pair correlation function in a DMC simulation, we use the expression

$$
g(r)=\frac{1}{\pi r h n N} \sum_{i<j} \nu_{h}\left(\left|\mathbf{r}_{i j}-\mathbf{r}\right|\right),
$$

where $v_{h}(r)=1$ if $r<h$ and zero otherwise. From this the probability of finding a boson at a distance $r$ away from an impurity as follows:

$$
g_{I B}(r)=\frac{1}{\pi r h n} \sum_{i<j} \nu_{h}\left(\left|\mathbf{r}_{i j}-\mathbf{r}\right|\right),
$$

where $v_{h}(r)=1$ if $r<h$ and zero otherwise. Pair-correlation function $g_{I B}(r)$ is displayed in Fig. 5 for $n a^{2}=10^{-5}$ and various values of $b / a$. Here, we use the healing length in $\overline{2 D} \xi=\sqrt{\ln \left(n a^{2}\right) / n^{41}}$ to scale the distance $r$. We find that the effect of strong impurity-boson interaction is reflected in a larger depletion at small $r$ and a peak structure developing around $r / \xi \sim 0.8$.

The density profile of the bosons surrounding the impurity can be calculated using the following integral of the impurity-boson pair-correlation function ${ }^{31}$ :

$$
n(r)=2 n \frac{\int_{0}^{r} d r^{\prime} r^{\prime} g_{I B}\left(r^{\prime}\right)}{r^{2}} .
$$

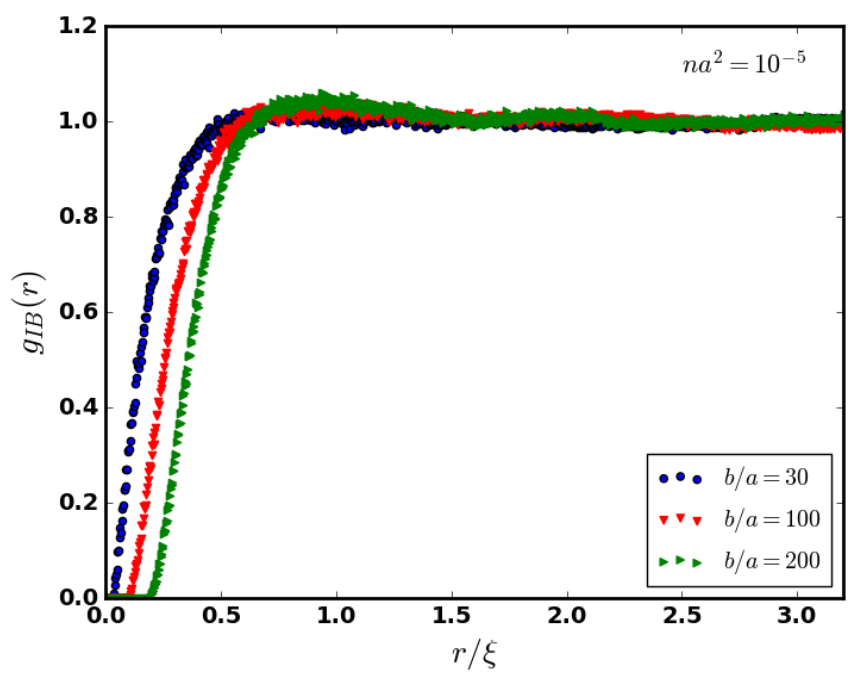

Fig. 5. (Color online) Impurity-boson pair correlation function $g_{I B}(r)$ as a function of $r / \xi$ for $n a^{2}=10^{-5}$ and different $b / a$ values. $g_{I B}(r)$ gives the probability of finding a boson a distance $r$ away from impurity. 


\section{E. Akaturk \& B. Tanatar}

We have calculated the above integral numerically using the pair correlation function $g_{I B}(r)$ from DMC simulations. Our results of $n(r)$ are shown in Fig. 6 as a function of $r / \xi$ for $n a^{2}=10^{-5}$ and various values of $b / a$. For large values of $r$, $n(r)$ tends to the boson density $n$. For small $r$, the strong impurity-boson repulsive interaction creates a "correlation hole" around the impurity much like the behavior in $g_{I B}(r)$. The small $r$ behavior of $n(r)$ in $2 \mathrm{D}$ is different from the results of Ardila

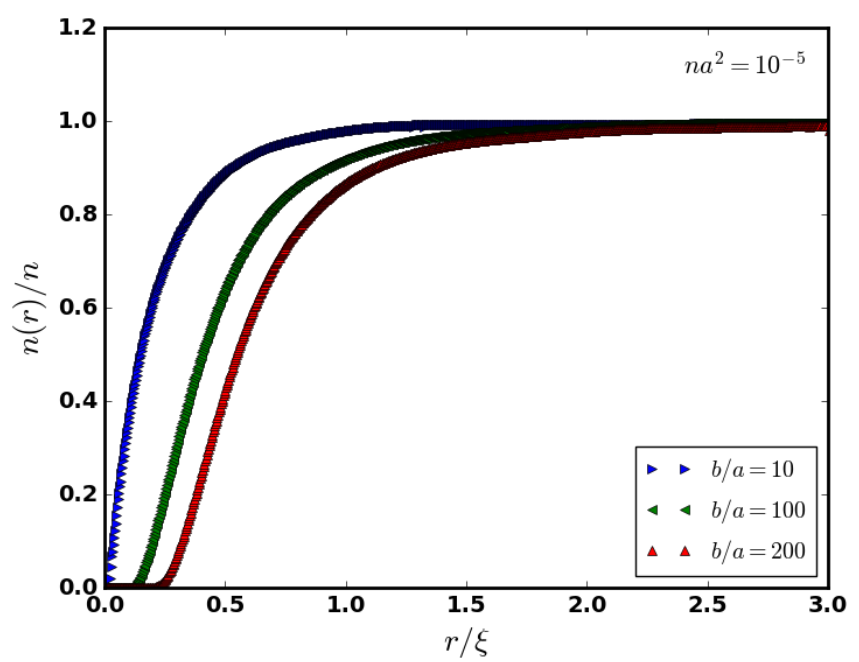

Fig. 6. (Color online) Ratio of local to uniform density of bosons $n(r) / n$ as a function of $r / \xi$ for $n a^{2}=10^{-5}$ and different $b / a$ values.

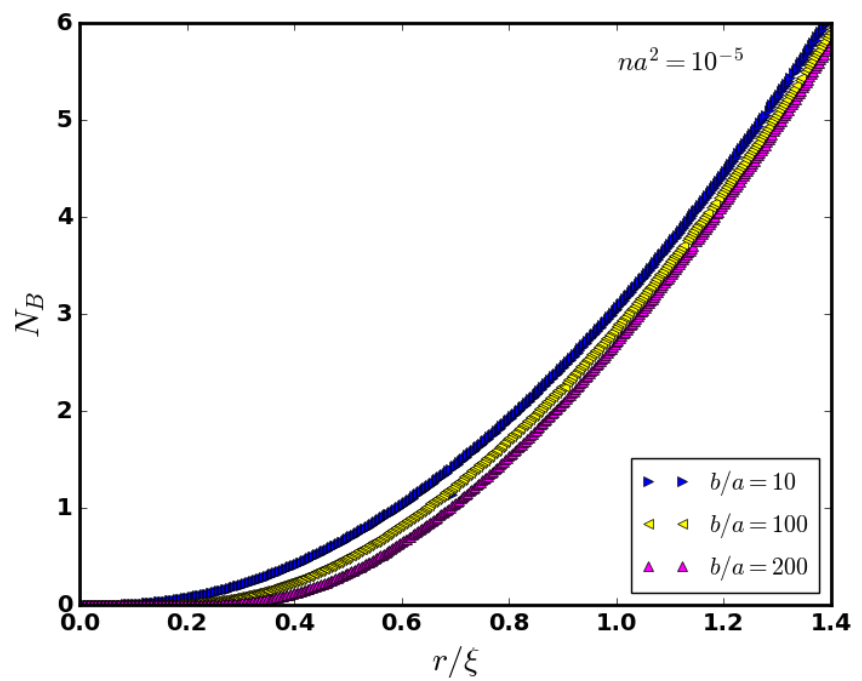

Fig. 7. (Color online) Average number of bosons $N_{B}$ surrounding the impurity as a function of $r / \xi$ for $n a^{2}=10^{-5}$ and different $b / a$ values. 
and Giorgini ${ }^{31}$ who have found a diverging behavior for $n(r)$ as $r \rightarrow 0$ in a 3D system. The reason for this is that they use a soft-well potential with an attractive part to model the impurity-boson interaction. In our case the interaction is purely repulsive. The average number of bosons $N_{B}$ surrounding the impurity is calculated by integrating the local density, $N_{B}=2 \pi \int_{0}^{r} d r^{\prime} r^{\prime} n\left(r^{\prime}\right)$ and shown in Fig. 7 . We find that $N_{B}$ increases with $r / \xi$, but the small $r$ behavior is largely determined by the ratio $b / a$. For a given density $n a^{2}$, as the impurity-boson interaction increases (large $b$ ) the value of one particle $\left(N_{B}=1\right)$ is reached at larger values of $r / \xi$.

\section{Concluding Remarks}

We have considered a mobile impurity in a system of bosons in 2D, all interacting repulsively, and performed QMC simulations at a very small value of the density parameter $n a^{2}$ and for various values of the ratio of boson-boson and impurity-boson scattering lengths $a / b$. We have found that at low densities and weak impurityboson interactions strengths perturbation theory adequately describes the polaron energy and effective mass. Our calculations discussed here may be extended in several directions. First, many impurities may be included in the simulations to study complete phase separation between the bosons and impurities, among other physical properties. Second, external trapping potentials may easily be taken into account in the simulations with appropriately chosen trial wavefunctions and Jastrow factors. Third, introducing a soft-well potential for the impurity-boson interaction may also be of interest since it would allow studying the excited states. The effects of mass difference between the impurity and bosons may also be probed. We hope our results reported here stimulates further work on Bose polarons, particularly experiments in lower dimensions.

\section{Acknowledgments}

We thank M. O. Oktel for valuable discussions and M. Holzmann for useful suggestions. This work is partially supported by TUBITAK and TUBA.

\section{References}

1. S. I. Pekar, Zh. Eksp. Teor. Fiz. 16, 341 (1946).

2. L. D. Landau and S. I. Pekar, Zh. Eksp. Teor. Fiz. 18, 419 (1948).

3. H. Fröhlich, Adv. Phys. 3, 325 (1954).

4. T. Holstein, Ann. Phys. 8, 325 (1959).

5. T. Holstein, Ann. Phys. 8, 343 (1959).

6. N. F. Mott, Physica C 205, 191 (1993).

7. S. Dzhumanov et al., Physica C 254, 311 (1995).

8. S. Dzhumanov, Superlattices Microstruct. 21, 363 (1997).

9. S. Ospelkaus et al., Phys. Rev. Lett. 96, 180403 (2006).

10. S. Schmid, A. Härter and J. H. Denschlag, Phys. Rev. Lett. 105, 133202 (2010).

11. C. Zipkes et al., Nature (London) 464, 388 (2010).

12. N. Spethmann et al., Phys. Rev. Lett. 109, 235301 (2012). 
13. N. B. Jørgensen et al., Phys. Rev. Lett. 117, 055302 (2016).

14. M.-G. Hu et al., Phys. Rev. Lett. 117, 055301, (2016).

15. F. Chevy and C. Mora, Rep. Prog. Phys. 73, 112401 (2010).

16. G. E. Astrakharchik and L. P. Pitaevskii, Phys. Rev. A 70, 013608 (2004).

17. R. M. Kalas and D. Blume, Phys. Rev. A 73, 043608 (2006).

18. F. M. Cucchietti and E. Timmermans, Phys. Rev. Lett. 96, 210401 (2006).

19. M. Bruderer, W. Bao and D. Jaksch, Europhys. Lett. 82, 30004 (2008).

20. D. H. Santamore and E. Timmermans, New J. Phys. 13, 103029 (2011).

21. A. A. Blinova, M. G. Boshier and E. Timmermans, Phys. Rev. A 88, 053610 (2013).

22. J. Tempere et al., Phys. Rev. B 80, 184504 (2009).

23. A. Novikov and M. Ovchinnikov, J. Phys. B: At. Mol. Opt. Phys. 43, 105301 (2010).

24. S. P. Rath and R. Schmidt, Phys. Rev. A 88, 053632 (2013).

25. A. G. Volosniev, H.-W. Hammer and N. T. Zinner, Phys. Rev. A 92, 023623 (2015).

26. W. Li and S. Das Sarma, Phys. Rev. A 90, 013618 (2014).

27. R. S. Christensen, J. Levinsen and G. M. Bruun, Phys. Rev. Lett. 115, 160401 (2015).

28. J. Levinsen, M. M. Parish and G. M. Bruun, Phys. Rev. Lett. 115, 125302 (2015).

29. F. Grusdt et al., Sci. Rep. 5, 12124 (2015).

30. V. Pastukhov, J. Phys. B: At. Mol. Opt. Phys. 51, 155203 (2018).

31. L. A. Peña Ardila and S. Giorgini, Phys. Rev. A 92, 033612 (2015).

32. L. A. Peña Ardila and S. Giorgini, Phys. Rev. A 94, 063640 (2016).

33. L. Parisi and S. Giorgini, Phys. Rev. A 95, 023619 (2017).

34. F. Grusdt, G. E. Astrakharchik and E. Demler, New J. Phys. 19, 103035 (2017).

35. K. Sacha and E. Timmermans, Phys. Rev. A 73, 063604 (2006).

36. C. Castelnovo, J. S. Caux and S. H. Simon, Phys. Rev. A 93, 013613 (2016).

37. L. A. Peña Ardila and T. Pohl, J. Phys. B: At. Mol. Opt. Phys. 52, 015004 (2019).

38. F. Grusdt and M. Fleischhauer, Phys. Rev. Lett. 116, 053602 (2016).

39. M. Schick, Phys. Rev. A 3, 1067 (1971).

40. D. F. Hines and N. E. Frankel, Phys. Lett. A 68, 12 (1978).

41. A. Posazhennikova, Rev. Mod. Phys. 78, 1111 (2006).

42. S. Pilati et al., Phys. Rev. A 71, 023605 (2005).

43. D. S. Petrov and G. E. Astrakharchik, Phys. Rev. Lett. 117, 100401 (2016).

44. G. E. Astrakharchik, Quantum Monte Carlo study of ultracold gases, PhD thesis, University of Trento, Italy (2004).

45. T. Pang, Am. J. Phys. 82, 980 (2014).

46. M. Boninsegni and D. M. Ceperley, Phys. Rev. Lett. 74, 2288 (1995).

47. J. Boronat and J. Casulleras, Phys. Rev. B 59, 8844 (1999). 